\title{
Thermodynamic perturbation theory for molecular liquid mixtures
}

Cite as: J. Chem. Phys. 78, 5750 (1983); https://doi.org/10.1063/1.445458

Submitted: 17 November 1982 . Accepted: 29 December 1982 . Published Online: 31 August 1998

Johann Fischer, and Santiago Lago

\section{ARTICLES YOU MAY BE INTERESTED IN}

Perturbation Theory and Equation of State for Fluids. II. A Successful Theory of Liquids

The Journal of Chemical Physics 47, 4714 (1967); https://doi.org/10.1063/1.1701689

Perturbation Theory and Equation of State for Fluids: The Square-Well Potential

The Journal of Chemical Physics 47, 2856 (1967); https://doi.org/10.1063/1.1712308

Equilibrium Thermodynamic Properties of the Mixture of Hard Spheres

The Journal of Chemical Physics 54, 1523 (1971); https://doi.org/10.1063/1.1675048
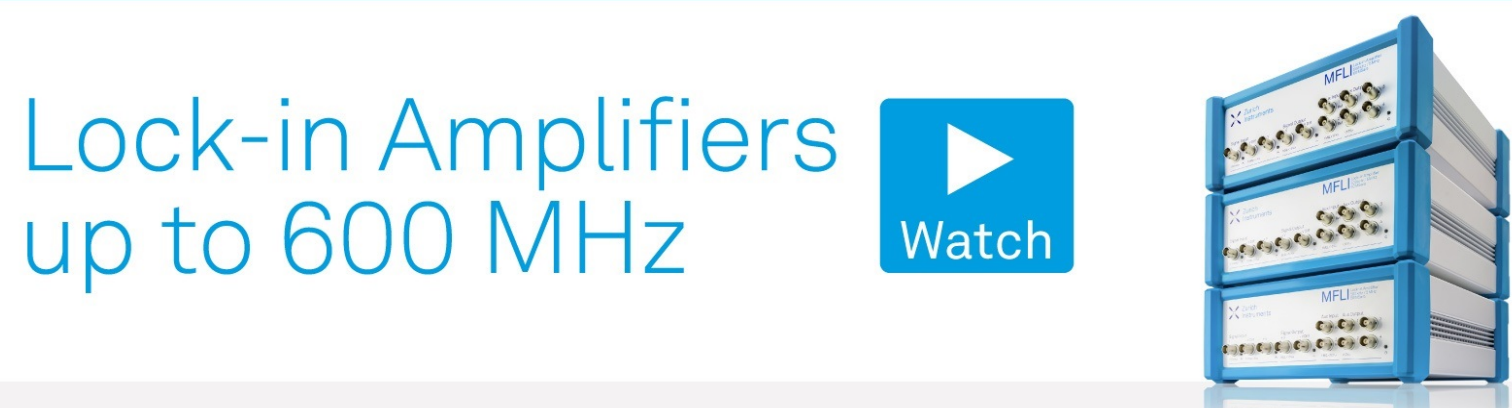

J. Chem. Phys. 78, 5750 (1983); https://doi.org/10.1063/1.445458

(c) 1983 American Institute of Physics. 


\title{
Thermodynamic perturbation theory for molecular liquid mixtures
}

\author{
Johann Fischer and Santiago Lago ${ }^{\text {a) }}$ \\ Institut für Thermo-und Fluiddynamik, Ruhr-Universität, D 4630 Bochum, Germany \\ [Received 17 November 1982; accepted 29 December 1982)
}

\begin{abstract}
A Weeks-Chandler-Andersen type perturbation theory for the Helmholtz energy of mixtures consisting of molecules with nonspherical cores is given. The correlation functions are obtained from a reference mixture of softly repulsive spherical particles. For that mixture the Percus-Yevick equation is solved with Baxter's formalism. By a blip expansion a hard convex body system is determined for which the free energy is obtained from Boublik's equation. For one-center Lennard-Jones liquids, the excess properties for mixtures agree with simulation results as good as those of the Baker-Henderson and the variational theory, while the pure substance properties are obtained better now. For mixtures of one-center and two-center Lennard-Jones liquids, the excess volumes and the excess enthalpies are given for argon/nitrogen and argon/oxygen after fitting the unlike pair interaction to the experimental value of the excess Gibbs energy. The results resemble those obtained from simpler theories, but discrepancies with respect to the reported experimental data remain.
\end{abstract}

\section{INTRODUCTION}

Reviews on mixtures of spherical molecules were given by Henderson and Leonard ${ }^{1}$ and by McDonald. ${ }^{2}$ Important contributions to the subject are the simulations of Singer and Singer ${ }^{3}$ and McDonald, ${ }^{4}$ the variational approach of Mansoori and Leland, ${ }^{5}$ a revised Barker-Henderson (BH) perturbation theory, ${ }^{6}$ and extensions of the Weeks-Chandler-Andersen ${ }^{7}$ (WCA) perturbation theory by Lee and Levesque ${ }^{8}$ and by Boublík. ${ }^{9}$ We learn that variational $1^{5}$ and $\mathrm{BH}$ theory ${ }^{6}$ yield excess properties which are in very good agreement with the simulation results. The WCA theories ${ }^{8.9}$ give better total properties. The excess properties, however, are somewhat scattered. The reason for that may be caused by extrapolation procedures for the background correlation functions. It is interesting to note that van der Waals one-fluid theory yields very good results as long as the sizes and the interaction energies of the molecules are not too different. ${ }^{2}$ The problem in predicting real substance properties, however, is with the combination rule for the unlike pair interactions. Using the

Lorentz-Berthelot rule can yield excess properties with even the wrong sign. ${ }^{2}$ To overcome that difficulty, other combination rules have been suggested. ${ }^{10,11}$ Application of Kohler's rule to liquid mixtures shows considerable improvement over the Lorentz-Berthelot results. ${ }^{12}$

For mixtures of nonspherical molecules, which are of greater practical importance, only a few statistical mechanical approaches not based on lattice theories have been published so far. For molecules with multipolar interactions the Padé approximant method ${ }^{13}$ has been applied by $\mathrm{Smith}^{14}$ and by Gubbins and co-workers. ${ }^{15}$ Although the method is based on heuristic arguments it yields good thermodynamic results. Presently, however, it is limited by the fact that only molecules with spherical cores can be treated. The only approach to molecules with nonspherical cores seems to be that of

\footnotetext{
a)Present address: Departamento Quimica Fisica and Instituto Rocasolano CSIC, Facultad Quimicas, Universidad Complutense, Madrid 3, Spain.
}

Enciso and Lombardero, ${ }^{16}$ who treat an interaction site model with Lennard-Jones interactions. They essentially apply Barker-Henderson perturbation theory calculating the site-site correlation functions from the RISM equation. ${ }^{17}$ It is, however, somewhat disappointing that the excess enthalpies are in bad agreement with experiment even if the combination rule has been fitted to the experimental value of the excess Gibbs energy. It may be that these effects are due to inaccuracies in the RISM solutions close to the contact values. ${ }^{18}$ Recent simulation results seem to confirm this point. ${ }^{19}$

In this paper we give an extension to liquid mixtures of a previous perturbation theory ${ }^{20,21}$ for pure molecular liquids in the formulation of Fischer's version. ${ }^{20}$ It is our intention to treat pure liquids and mixtures of spherical and nonspherical molecules within the same approximation scheme with good accuracy.

In Sec. II we give a detailed description of the theory for a mixture of one-center Lennard-Jones (1CLJ) and homonuclear two-center Lennard-Jones (2CLJ) molecules. Essentially, we start by dividing the potential in the sense of WCA. The correlation functions for the reference system of softly repulsive nonspherical particles are obtained by going back to softly repulsive spherical particles, which are introduced by angle averaging over the Boltzmann factors of the reference system. For that mixture of spherical particles we solve the Percus-Yevick equation ${ }^{22}$ in the framework of Baxter's formalism. ${ }^{23}$ Details of the numerical procedure are given in Appendix A. Having thus constructed the correlation functions we look for a corresponding mixture of hard spheres and hard dumbbells by a blip expansion. For the latter mixture the Helmholtz energy can be obtained from Boublík's equation. ${ }^{24}$ Details concerning the calculation of the blip integrals are described in Appendix B.

The application of the theory to some other mixtures consisting of symmetric site-site molecules is straightforward, e.g., to a mixture of $1 \mathrm{CLJ}$ molecules or to a 
mixture of homonuclear 2CLJ molecules. We think that the basic ideas of the theory can be successfully used for a much larger class of interactions. Then, however, additional approximations are necessary.

In Sec. III we present results: (a) For a pure $1 \mathrm{CLJ}$ liquid we compare zero pressure densities and Helmholtz energies with simulation results and those obtained from the Verlet-Weiss-Grundke-Henderson (VWGH) version $^{25}$ of the WCA approach. (b) For 1CLJ mixtures we compare excess properties with simulation and other theoretical results. (c) Mixtures of $1 \mathrm{CLJ}$ and 2CLJ molecules corresponding to argon/nitrogen and argon/ oxygen are considered using the Lorentz-Berthelot and an adjusted combining rule. Moreover, correlation functions are also shown for that case.

\section{THEORY}

The binary mixture is considered at temperature $T$, particle density $\rho$, and mole fractions $x_{A}$ and $x_{B}$. Component $A$ consists of $1 \mathrm{CLJ}$ molecules, component $\mathrm{B}$ of homonuclear 2CLJ molecules. The total interaction energy between any two molecules shall be $u_{A A}, u_{A B}$, and $u_{B B}$, respectively. The energy $u_{A A}$ is simply a $12 / 6$ Lennard-Jones (LJ) interaction with parameters $\epsilon_{A A}$ and $\sigma_{\mathrm{AA}}$. The energy $u_{\mathrm{BB}}$ is composed of four equal $\mathrm{LJ}$ interactions with parameters $\epsilon_{\mathrm{BB}}$ and $\sigma_{\mathrm{BB}}$ between any two interaction sites on two different $B$ molecules, the interaction sites on each molecule being a distance $l$ apart. Finally, the energy $u_{\mathrm{AB}}$ consists of two equal $\mathrm{L}_{\mathrm{J}} \mathrm{J}$ interactions with parameters $\epsilon_{\mathrm{AB}}$ and $\sigma_{\mathrm{AB}}$ between the center of an A molecule and each site on a $B$ molecule. The interaction sites on the $B$ molecules shall be the same for the $A-B$ interaction as for the $B-B$ interaction. As the potentials $u_{\alpha \beta}$ for $\alpha, \beta=\mathrm{A}, \mathrm{B}$ depend on the distance $r$ between the centers and eventually on the orientations $\omega_{1}$ and $\omega_{2}$ of the molecules, we write $u_{\alpha \beta}\left(r, \omega_{1}, \omega_{2}\right)$. Moreover, it is useful to relate the interaction parameters by

$$
\begin{aligned}
& \epsilon_{\mathrm{AB}}=\xi\left(\epsilon_{\mathrm{AA}} \epsilon_{\mathrm{BB}}\right)^{1 / 2}, \\
& \sigma_{\mathrm{AB}}=\eta\left(\sigma_{A A}+\sigma_{B B}\right) / 2 .
\end{aligned}
$$

The case $\xi=\eta=1$ shall be termed as Lorentz-Berthelot combination rule.

As the first step in perturbation theory we introduce a reference system of "softly repulsive," nonspherical molecules. For that purpose we consider each pair potential $u_{\alpha \beta}\left(r, \omega_{1}, \omega_{2}\right),(\alpha, \beta=\mathrm{A}, \mathrm{B})$ for a fixed mutual orientation $\left(\omega_{1}, \omega_{2}\right)$ of the molecules as a function of the distance $r$ and cut it at its minimum. Let $r_{\alpha \beta, m i n}\left(\omega_{1}, \omega_{2}\right)$ be the position of the minimum and $u_{\alpha \beta, m i n}\left(\omega_{1}, \omega_{2}\right)$ its value, then the potentials $u_{\alpha \beta}^{0}$ of the reference system are

$$
\begin{array}{ll}
u_{\alpha \beta}^{0}\left(r, \omega_{1}, \omega_{2}\right)=u_{\alpha \beta}\left(r, \omega_{1}, \omega_{2}\right)+u_{\alpha \beta, \mathrm{min}}\left(\omega_{1}, \omega_{2}\right) \\
u_{\alpha \beta}^{0}\left(r, \omega_{1}, \omega_{2}\right)=0 & \text { for } r<r_{\alpha \beta, \mathrm{min}}\left(\omega_{1}, \omega_{2}\right),
\end{array}
$$

Hence, the perturbation potentials are

$$
u_{\alpha \beta}^{1}=u_{\alpha \beta}-u_{\alpha \beta}^{0} \text {. }
$$

It has been discussed in our previous work $\mathrm{k}^{20}$ why this choice of the reference system is better than simply cutting the site-site interactions at their minima. For $u_{\mathrm{AB}}$ and $u_{\mathrm{BB}}$ the division of the potential is done numerically.

Having thus divided the potentials into reference and perturbation parts, the configurational partition function can be expanded which yields for the Helmholtz energy to first order ${ }^{25}$

$$
A=A^{0}+(N \rho / 2) \sum_{\alpha, \beta} x_{\alpha} x_{\beta} A_{\alpha \beta}^{1},
$$

with

$$
A_{\alpha \beta}^{1}=\int u_{\alpha \beta}^{1}\left(r, \omega_{1}, \omega_{2}\right) g_{\alpha \beta}^{0}\left(r, \omega_{1}, \omega_{2}\right) d \omega_{1} d \omega_{2} d \mathbf{r} .
$$

Here $A^{0}$ is the Helmholtz energy of the reference system consisting of softly repulsive nonspherical molecules with interactions $u_{\alpha \beta}^{0}$. The $g_{\alpha \beta}^{0}$ are the pair correlation functions of that reference system.

The pair correlation functions $g_{\alpha \beta}^{0}$ are needed for evaluating the $A_{\alpha \beta}^{1}$ terms and for finding a hard body system with approximately the same free energy as $A^{0}$ by a blip expansion. We obtain them as zeroth order terms of a perturbation expansion ${ }^{26}$ in which softly repulsive spherical potentials $\Phi_{\alpha \beta}$ are introduced by

$$
\exp \left[-\beta \Phi_{\alpha \beta}(\gamma)\right]=\left\langle\exp \left[-\beta u_{\alpha \beta}^{0}\left(r, \omega_{1}, \omega_{2}\right)\right]\right\rangle,
$$

where the angular brackets denote angle-averaging over the orientations $\omega_{1}$ and $\omega_{2}$, if $u_{\alpha \beta}^{0}$ depends on them, and $\beta=1 / k T$. For the mixture of spherical particles interacting with the potentials $\Phi_{\alpha \beta}$ the pair correlation functions $\hat{g}_{\alpha \beta}$ and the background correlation functions $\hat{y}_{\alpha \beta}$ $=\exp \left(\beta \Phi_{\alpha \beta}\right) \hat{g}_{\alpha \beta}$ are determined by solving the PercusYevick equation in Baxter's formulation. ${ }^{23}$ The numerical procedure is described in Appendix A. Then the pair correlation functions $g_{\alpha \beta}^{0}$ of the reference system are approximated ${ }^{26}$ as

$$
g_{\alpha \beta}^{0}\left(r, \omega_{1}, \omega_{2}\right)=\exp \left[-\beta u_{\alpha \beta}^{0}\left(r, \omega_{1}, \omega_{2}\right)\right] \hat{y}_{\alpha \beta}(r) .
$$

The Helmholtz energy $A^{0}$ of the reference system is determined with the help of a mixture of hard spheres and hard dumbbells, the latter having the same distance $l$ between the interaction sites as the $2 \mathrm{CLJ}$ molecules. For that purpose we use a blip expansion ${ }^{20}$ of the Helmholtz energy $A^{H}$ of the hard system around $A^{0}$. Let us introduce the abbreviations

$$
\begin{aligned}
& e_{\alpha \beta}^{0}=\exp \left[-\beta u_{\alpha \beta}^{0}\left(r, \omega_{1}, \omega_{2}\right)\right], \\
& e_{\alpha \beta}^{H}=\exp \left[-\beta u_{\alpha \beta}^{H}\left(r, \omega_{1}, \omega_{2}\right)\right],
\end{aligned}
$$

where the $u_{\alpha \beta}^{H}$ denote the potentials between the hard spheres and hard dumbbells, then the functional expansion is done in terms of $\Delta e_{\alpha \beta}=e_{\alpha \beta}^{H}-e_{\alpha \beta}^{0}$. This yields to first order

$$
A^{H} / N k T=A^{0} / N k T-(\rho / 2) \sum_{\alpha \beta \beta} x_{\alpha} x_{\beta} B_{\alpha \beta},
$$

with

$$
B_{\alpha \beta}=\int\left(e_{\alpha \beta}^{H}-e_{\alpha \beta}^{0}\right) y_{\alpha \beta}^{0} d \omega_{1} d \omega_{2} d \mathbf{r} .
$$

Using the approximation $y_{\alpha \beta}^{0}=\hat{y}_{\alpha \beta}$, expressed by Eq. (8), the blip integrals $B_{\alpha \beta}$ can be approximated by 
TABLE I. Densities and residual Helmholtz energies at zero pressure for a 1CLJ liquid. Results of perturbation theories are compared with simulation data of McDonald and Singer ${ }^{2}$ and Adams. ${ }^{b}$ Column two gives the zero pressure densities obtained by direct interpolation on the Monte Carlo isotherms while column three gives the values from the fitted equation of state (EOS).

\begin{tabular}{|c|c|c|c|c|c|c|c|}
\hline \multirow[b]{2}{*}{$k T / \epsilon$} & \multicolumn{3}{|c|}{ Simulation } & \multicolumn{2}{|c|}{ WCA/VWGH } & \multicolumn{2}{|c|}{ This work } \\
\hline & $\rho \sigma^{3}$ (direct) & $\rho \sigma^{3}(\mathrm{EOS})$ & $A_{r} / N k T$ (EOS) & $\rho \sigma^{3}$ & $A_{r} / N k T$ & $\rho \sigma^{3}$ & $A_{r} / N k T$ \\
\hline 0.720 & 0.838 & 0.842 & -4.93 & 0.840 & -4.86 & 0.831 & -4.80 \\
\hline 0.810 & 0.799 & 0.799 & -3.97 & 0.803 & -3.90 & 0.794 & -3.85 \\
\hline 0.902 & 0.757 & 0.751 & -3.21 & 0.762 & -3.15 & 0.754 & -3.11 \\
\hline 0.977 & 0.714 & 0.710 & -2.72 & 0.726 & -2.66 & 0.719 & -2.62 \\
\hline
\end{tabular}

${ }^{\mathrm{a}}$ Reference 29.

Beference 30.

$$
B_{\alpha \beta}=\int\left(\left\langle e_{\alpha \beta}^{H}\right\rangle-\left\langle e_{\alpha \beta}^{0}\right\rangle\right) \hat{y}_{\alpha \beta} d \mathbf{r}
$$

In order to determine the three hard interaction diameters $d_{\mathrm{AA}}, d_{\mathrm{AB}}$, and $d_{\mathrm{BB}}$, the procedure is not unique now. Similarly to the treatment of spherical mixtures by other authors ${ }^{8,9}$ we chose $d_{\mathrm{AA}}$ and $d_{\mathrm{BB}}$ by

$$
B_{A \mathrm{~A}}=0, B_{\mathrm{BB}}=0 \text {. }
$$

Then we fixed

$$
d_{\mathrm{AB}}=\left(d_{\mathrm{AA}}+d_{\mathrm{BB}}\right) / 2,
$$

as the hard convex body equation of Boublik ${ }^{24}$ is valid only for additive hard interactions. Thus, Eq. (10) finally yields

$$
\begin{aligned}
A^{0} / N k T= & A^{H} / N k T+\rho x_{\mathrm{A}} x_{\mathrm{B}} \\
& \times \int\left(\left\langle e_{\mathrm{AB}}^{H}\right\rangle-\left\langle e_{\mathrm{AB}}^{0}\right\rangle\right) \hat{y}_{\mathrm{AB}} d \mathbf{r} .
\end{aligned}
$$

In spite of the fact that the correction term represented by the integral is generally rather small it cannot be neglected in the calculation of the excess properties. The numerical treatment of the blip integrals $B_{\alpha \beta}$ in the approximation of Eq. (12) is described in Appendix B.

The Helmholtz energy $A^{H}$ of the mixture of hard spheres and hard dumbbells is obtained from Boublik's hard convex body equation of state. ${ }^{24}$ Consider a hard convex body $\alpha$ and let $V_{\alpha}$ be the volume, $S_{\alpha}$ the surface, $R_{\alpha}$ the $(1 / 4 \pi)$ multiple of the mean curvature integral, and $Q_{\alpha}=R_{\alpha}^{2}$. For the description of the mixture "one fluid" quantities are introduced by $A=\Sigma x_{\alpha} A_{\alpha}$, where $A$ stands for any of the geometric quantities $V, S, R$, or $Q$. With the help of the dimensionless quantities $k_{1}$ $=(R S / V)$ and $k_{2}=\left(Q S^{2} / 9 V^{2}\right)$ the equation of state of the hard convex body mixture writes as

$$
\frac{p}{\rho k T}=\frac{1}{1-v}+\frac{k_{1} v}{(1-v)^{2}}+\frac{k_{2} v^{2}(3-v)}{(1-v)^{3}},
$$

where $v=\rho V$. Equation (16) can be integrated to give for the residual part $A_{r}^{H}$ of the Helmholtz energy

$$
\begin{aligned}
A_{r}^{H} / N k T= & \left(k_{2}-1\right) \ln (1-v) \\
& +\left[k_{1}+k_{2}-k_{1} v\right] v /(1-v)^{2} .
\end{aligned}
$$

In a later paper ${ }^{27}$ it was shown that this equation also yields good results for a system of hard dumbbells if the mean curvature integral is taken as that of a prolate spherocylinder. Thus, the required geometric quanti- ties of a hard dumbbell with sphere diameter $d$ and a center-to-center distance $l$ are

$$
\begin{aligned}
& V_{D}=(\pi / 6) d^{3}\left(1+3 / 2 L-1 / 2 L^{3}\right), \\
& S_{D}=\pi d^{2}(1+L), \\
& R_{D}=(1 / 2) d(1+1 / 2 L),
\end{aligned}
$$

with $L=l / d$,

\section{RESULTS}

The intention is to show the accuracy of our perturbation theory for different model liquids. This is done by comparison with simulation data and other theoretical results, where possible. Since for mixtures of $1 \mathrm{CLJ}$ and 2CLJ molecules only preliminary simulation results are available ${ }^{19}$ we compare with experimental data. It shall be mentioned that for pure 2CLJ liquids the comparison has already been given in the previous paper on perturbation theory. ${ }^{20}$

For predicting the excess properties of real liquid mixtures the combination rule is crucial. This point shall be deferred to a later paper. ${ }^{12}$

\section{A. The pure 1CLJ liquid}

The WCA/VWGH theory ${ }^{25}$ is considered as one of the most successful perturbation theories for that case. It uses a hard sphere reference system and correlation functions which have been corrected to agree with simulation results. In our case the reference system consists of soft repulsive spheres and the correlation functions are the PY solutions for that reference system. To check the accuracy of our approach we compare in Table I densities and Helmholtz energies at zero pressure with results of simulations and of the WCA/VWGH theory. These quantities are important for calculating excess functions of mixtures. The WCA/VWGH results have been computed by the present authors.

Some words concerning the simulation results seem to be necessary. At low temperatures $p-p-T$ data of Verlet $^{28}$ and of McDonald and Singer ${ }^{29}$ are available. The latter have the advantage that an ansatz for the Helmholtz energy has been fitted to them and the absolute value of the free energy has been calculated consistently with this ansatz by Adams ${ }^{30}$ using a grand canonical ensemble. Therefore, we used these results for comparison. The zero pressure densities can be obtained either 
TABLE II. Excess properties obtained from simulations and different theories for equimolar model mixtures of $1 \mathrm{CLJ}$ liquids at zero pressure. The interaction parameters for the pure substances are listed in Ref. 2, for the crossed interactions the Lorentz-Berthelot rule is used. $g^{E}$ and $h^{E}$ are given in $\mathrm{J} / \mathrm{mol}, v^{E}$ in $\mathrm{cm}^{3} / \mathrm{mol}$.

\begin{tabular}{|c|c|c|c|c|c|c|c|c|c|}
\hline & \multicolumn{3}{|c|}{$\mathrm{Ar} / \mathrm{Kr}(116 \mathrm{~K})$} & \multicolumn{3}{|c|}{$\mathrm{Ar} / \mathrm{N}_{2}(84 \mathrm{~K})$} & \multicolumn{3}{|c|}{$\mathrm{Ar} / \mathrm{CH}_{4}(91 \mathrm{~K})$} \\
\hline & $g^{E}$ & $h^{E}$ & $v^{E}$ & $g^{E}$ & $h^{E}$ & $v^{E}$ & $g^{E}$ & $h^{E}$ & $v^{E}$ \\
\hline$\overline{\mathrm{MC}-N, v, T^{\mathrm{a}}}$ & 45 & -18 & -0.60 & 35 & $\overline{40}$ & -0.23 & -10 & -35 & -0.12 \\
\hline $\mathrm{MC}-N, p, T^{\mathrm{b}}$ & 46 & -29 & -0.69 & 35 & 16 & -0.25 & -14 & -60 & -0.22 \\
\hline $\operatorname{Var}^{b, c}$ & 47 & -31 & -0.73 & 42 & 42 & -0.26 & -12 & -34 & -0.14 \\
\hline $\mathrm{BH}^{\mathrm{d}}$ & 39 & -28 & -0.62 & 32 & 35 & -0.23 & -12 & -30 & -0.12 \\
\hline This work & 40 & -19 & -0.51 & 35 & 40 & -0.22 & -13 & -33 & -0.12 \\
\hline WCA $/ L L^{e}$ & 36 & 21 & -0.48 & 40 & 56 & -0.26 & 4 & 41 & -0.12 \\
\hline $\mathrm{WCA} / \mathrm{B}^{\mathrm{f}}$ & 28 & -10 & -0.46 & & & & & & \\
\hline
\end{tabular}

by interpolation on the directly calculated isotherms or from the fitted equation of state. In spite of the high accuracy of that work the differences are remarkable with respect to the excess volumes of mixtures. Comparing the results of the different simulations shows that Verlet's zero pressure densities are generally smaller by about 0.010 in $\rho \sigma^{3}$ than those of McDonald and Singer. Some scattering is also in the Helmholtz energies obtained from different approaches. For the state point $k T / \epsilon=0.75$ and $\rho \sigma^{3}=0.84$ Hansen and Verlet $^{31}$ give $A_{r} / N k T=-4.53$, while from McDonald, Singer, and Adams one gets $A_{r} / N k T=-4.59$.

Both perturbation theories yield equally good results for the zero pressure densities. Their variation with temperature, however, seems to be slightly too weak. The Helmholtz energies seem to be better predicted by WCA/VWGH. Fortunately, the differences in $A_{r}$ obtained from the three approaches are nearly constant with temperature.

\section{B. Mixtures of $1 \mathrm{CLJ}$ liquids}

As it has been shown ${ }^{25}$ that the WCA theory is more accurate than the variational theory and the first order $\mathrm{BH}$ theory for pure liquids, it looks somewhat puzzling that the WCA results for the excess properties of mixtures reported hitherto ${ }^{8,9}$ are worse than the results of the two other approaches. ${ }^{5,6}$ We will demonstrate that this is not the case for our version of the WCA theory.

Monte Carlo studies have been made by Singer and Singer ${ }^{3}$ in the $N, v, T$ ensemble for molecules with size ratios $0.79<\sigma_{B B} / \sigma_{A A}<1.27$ and energy ratios $1<\epsilon_{B B} / \epsilon_{A A}$ $<1.52$. After some extrapolation processes these authors obtained the excess properties $g^{E}, v^{E}$, and $h^{E}$ at zero pressure for several model mixtures. As far as the errors are concerned in that procedure, the authors report standard deviations in the zero pressure volumes of the mixtures of 0.05 to $0.10 \mathrm{~cm}^{3} / \mathrm{mol}$. To avoid these extrapolation processes, simulations have been made by McDonald in the $N, p, T$ ensemble. ${ }^{4}$ The agreement between both simulation series is reasonable for $g^{E}$ and $v^{E}$, but not so for $h^{E}$. In Table II we compare these simulation results with the results of the variational theory, ${ }^{5,4}$ the BH-perturbation theory, ${ }^{6}$ the WCA theory of Lee and Levesque, ${ }^{8}$ the WCA theory of Boub- lik, ${ }^{9}$ and our own perturbation theory for the model mixtures $\mathrm{Ar} / \mathrm{Kr}, \mathrm{Ar} / \mathrm{N}_{2}(1 \mathrm{CLJ})$, and $\mathrm{Ar} / \mathrm{CH}_{4}$ (1CLJ).

We learn from Table II that the excess properties obtained from variational theory, Barker-Henderson perturbation theory and our WCA-type theory are in close agreement with the simulation data of Singer and Singer. The uncertainties in the simulation results do not allow discernment of the best approach. One problem with the WCA approaches of Lee and Levesque ${ }^{8}$ and of Boublik ${ }^{9}$ is presumably the fact that in the blip expansion the hard sphere correlations have to be known for distances smaller than the contact value. For those distances questionable extrapolation processes have been used in both cases. This extrapolation is not necessary in our formalism as the correlation functions are calculated for the softly repulsive spheres. In Boublikk's approach an additional source of error may be the fact that the hard-sphere diameters in the mixture are assumed to be the same as in the pure liquid. In the work of Lee and Levesque a potential inconsistency is in the fact that the pure substance properties are not calculated with perturbation theory but are obtained from a fitted equation of state.

Recently the umbrella sampling technique has been applied by Nakanishi et al. ${ }^{32}$ for the calculation of the excess Helmholtz energy of a mixture consisting of molecules of equal size but different energy interactions. Fortunately those authors already gave the results of variational theory for their model mixtures. In Table III we have added the results of our perturbation theory.

TABLE III. Excess Helmholtz energies $a^{E}$ for model mixtures of LB2, LB3, and LB4 type. The models are defined in Ref. 32. The state points are $x_{A}$ $=0.5, T=120 \mathrm{~K}$, and $\rho \sigma^{3}=0.75 ; a^{E}$ is given in $\mathrm{J} / \mathrm{mol}$.

\begin{tabular}{lccc}
\hline \hline & LB2 & LB3 & LB4 \\
\hline MC $^{\text {a }}$ & 174 & 438 & $\mathbf{7 6 7}$ \\
Variational $^{\mathrm{a}}$ & 187 & 476 & $\mathbf{7 6 9}$ \\
This work & $\mathbf{1 8 5}$ & 472 & $\mathbf{7 6 3}$ \\
\hline
\end{tabular}

${ }^{\mathrm{a}}$ Reference 32 . 
TABLE IV. Model parameters of argon, nitrogen, and oxygen and pure substance properties at $84 \mathrm{~K}$. The experimental values are from Refs. $34-36$.

\begin{tabular}{|c|c|c|c|c|c|c|}
\hline & \multicolumn{4}{|c|}{ Model 1CLJ/2CLJ } & \multicolumn{2}{|c|}{$\begin{array}{c}\text { Experimental } \\
\text { values }\end{array}$} \\
\hline & $\epsilon / k(\mathbf{K})$ & $\sigma(\AA)$ & $l(\AA)$ & $\begin{array}{c}v(p=0) \\
\left(\mathrm{cm}^{3} / \mathrm{mol}\right)\end{array}$ & $\begin{array}{c}v\left(p_{0}\right) \\
\left(\mathrm{cm}^{3} / \mathrm{mol}\right)\end{array}$ & $p_{\sigma}($ bar $)$ \\
\hline Ar & 119.8 & 3.405 & 0 & 28.34 & 28.26 & 0.705 \\
\hline $\mathrm{N}_{2}$ & 37.3 & 3.310 & 1.0897 & 35.85 & 36.06 & 2.077 \\
\hline $\mathrm{O}_{2}$ & 44.6 & 3.090 & 1.0166 & 27.16 & 27.30 & 0.504 \\
\hline
\end{tabular}

We observe that the results of both theories are close together in all three models and agree very well with the simulation results in the LB2 and LB4 model. The discrepancy in the LB3 model is thought to be within the accuracy of umbrella sampling.

Concluding we can say that for mixtures of spherical molecules our WCA-type perturbation theory yields excess properties which agree, even for strong energy differences, with those of the simulations within the accuracy of the latter. The difference, however, is in the prediction of the total properties, as for pure substances our theory is close to WCA/VWGH and hence considerably better than the two other approaches.

\section{Mixtures of $1 \mathrm{CLJ} / 2 \mathrm{CLJ}$ liquids}

For mixtures of molecules with nonspherical cores a BH-type perturbation theory was published ${ }^{16}$ in which the site-site correlation functions are calculated by RISM. That theory has been applied to $1 \mathrm{CLJ} / 2 \mathrm{CLJ}$ mixtures. For the lack of simulation results comparison was made with the experimental data of $\mathrm{Ar} / \mathrm{N}_{2}$ and $\mathrm{Ar} / \mathrm{O}_{2}$. We have applied our theory to the same mixtures and used the same pure substance parameters. ${ }^{33}$ We also performed calculations with the LorentzBerthelot combination rule and a value of $\xi \neq 1$ which was adjusted such that $g^{E}$ agrees with the experimental results.

In Table IV it is shown that the model parameters for argon, nitrogen, and oxygen yield zero pressure volumes which are in good agreement with the experimental data.
Experimental values for $g^{E}, h^{E}$, and $v^{E}$ of argon/ nitrogen ${ }^{37-39}$ and argon/oxygen ${ }^{37,39}$ have been critically selected by Rowlinson and Swinton. ${ }^{40}$ These authors also give a comparison with three simpler theories, the $\mathrm{BH}$-perturbation theory for $1 \mathrm{CLJ} / 1 \mathrm{CLJ}$ liquids, ${ }^{6}$ the one-fluid van der Waals theory, ${ }^{2,41}$ and the treatment of Snider and Herrington. ${ }^{2,42}$ In all theories the crossed interaction was fitted to the experimental $g^{E}$; hence $h^{E}$ and $v^{E}$ are a measure for the predictive power of the approach. Rowlinson and Swinton conclude that the results from all three theories resemble each other more closely than they agree with experiment. In Table $V$ we have extended that comparison by including the results of our perturbation theory and those of Enciso and Lombardero.

It is seen from Table $\mathrm{V}$ that the results of the BH/ RISM theory of Enciso and Lombardero are in strong disagreement with the experiments as well as with the other theories. Moreover it is physically hard to understand why the volume of the argon/oxygen mixture should decrease with decreasing $\xi$. The present authors believe that some inaccuracies in the numerical treatment could be the reason. Possibly the site-site correlation functions close to the contact points are in error. ${ }^{18,19}$

As to our results, we learn from Table $V$ that for argon/nitrogen $v^{E}$ is in good agreement with experiment, but not $h^{E}$. On the contrary, for argon oxygen $h^{E}$ is in good agreement, but not $v^{E}$. On the basis of these results a simultaneous variation of the parameters $\xi$ and $\eta$ in the combination rule could not improve the agreement. Comparing with the simpler theoretical approaches as given in Table $V$ or in Ref. 40 we can only repeat the statement of Rowlinson and Swinton that the results of all theories, ours included, resemble each other more closely than they agree with experiment. This is somewhat striking since it was thought ${ }^{40}$ that treating oxygen and nitrogen as nonspherical molecules would bring theory and experiment closer together.

One might speculate now about the persistent discrepancies between theory and experiment. Of course, there are several assumptions in the theories which could be the reason. On the other hand, some of the

TABLE V. Excess properties for the equimolar mixtures argon/nitrogen and argon/oxygen at $84 \mathrm{~K}$ and zero pressure. Experimental data are compared with the results for 1 CLJ/1CLJ or $1 \mathrm{CLJ} / 2 \mathrm{CLJ}$ model mixtures. In the theories the Lorentz-Berthelot and an adjusted combining rule are used. $g^{E}$ and $h^{E}$ are given in $\mathrm{J} / \mathrm{mol}, v^{E}$ in $\mathrm{cm}^{3} / \mathrm{mol}$.

\begin{tabular}{|c|c|c|c|c|c|c|c|c|}
\hline & \multicolumn{4}{|c|}{ Argon/nitrogen } & \multicolumn{4}{|c|}{ Argon/oxygen } \\
\hline & $\xi$ & $g^{E}$ & $h^{E}$ & $v^{E}$ & $\xi$ & $g^{E}$ & $h^{E}$ & $v^{E}$ \\
\hline$\overline{\operatorname{Expt}}{ }^{a}$ & & 34 & 52 & -0.18 & & 37 & 60 & 0.14 \\
\hline 1CLJ/1CLJ\} & 1.000 & 36 & 39 & -0.26 & 1.000 & 0 & 0 & 0.00 \\
\hline $\left.\mathrm{BH}^{\mathrm{b}}\right\}$ & 1.001 & 34 & 35 & -0.27 & 0.987 & 37 & 52 & 0.06 \\
\hline $1 \mathrm{CLJ} / 2 \mathrm{CLJ}\}$ & 1.000 & 27 & 20 & -0.18 & 1.000 & -29 & -41 & -0.09 \\
\hline This work & 0.9975 & 34 & 31 & -0.17 & 0.9797 & 37 & 56 & 0.03 \\
\hline $1 \mathrm{CLJ} / 2 \mathrm{CLJ}\}$ & 1.000 & 27 & -4 & -0.26 & 1.000 & -13 & $\cdots$ & 0.15 \\
\hline$E L^{c}$ & 0.997 & 34 & 8 & -0.24 & 0.983 & 37 & 14 & -0.05 \\
\hline
\end{tabular}




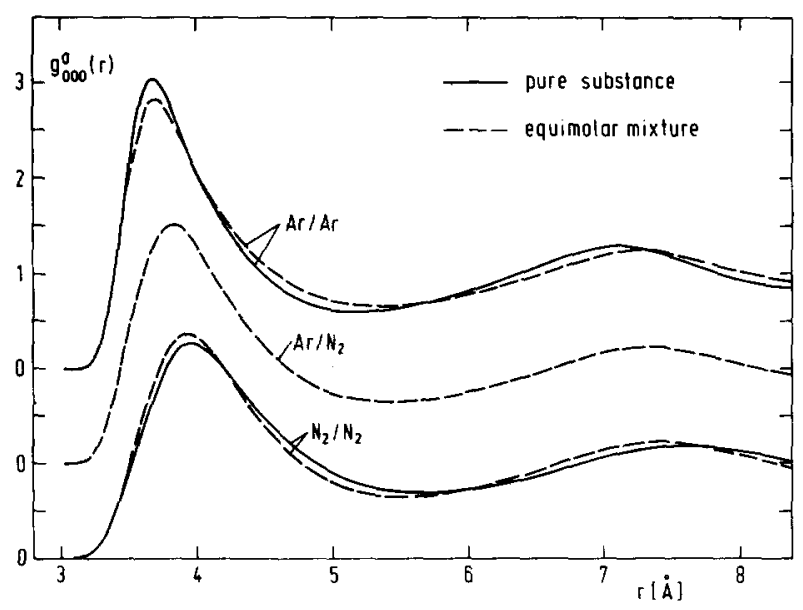

FIG. 1. Angle-averaged pair correlation functions $g_{000}^{0}$ of the softly repulsive reference systems for the model liquids argon (1CLJ), nitrogen (2CLJ), and their equimolar mixture at $84 \mathrm{~K}$ and zero pressure.

experimental data have only been measured once and some time ago, or have been measured twice by the same group. Considering then, that for krypton/xenon the experimental values for $v^{E}$ differ ${ }^{40}$ by about 0.20 $\mathrm{cm}^{3} / \mathrm{mol}$, our theoretical results could also be within the error bars of experiment.

Finally, Fig. 1 shows pair correlation functions $g_{000}^{0}$ for argon/nitrogen at $84 \mathrm{~K}$, zero pressure and $\xi$ $=0.9975$. According to our approach these are the angle-averaged correlation functions of the softly repulsive particles. We see that the correlation functions do not change significantly in going from the pure substances to the mixture. The results may be interesting for the justification of the one-fluid van der Waals model.

\section{ACKNOWLEDGMENTS}

The authors gratefully acknowledge fruitful discussions with Professor F. Kohler, Professor W. A. Steele, and Dr. G. Götze. A correspondence with Professor W. J. Meath from the University of Western Ontario, Canada, has contributed much to the improvement of the numerical accuracy. One of us (S.L.) wishes to thank the Humboldt Foundation for a fellowship. Part of the work was supported by the Deutsche Forschungsgemeinschaft, project number Fi 287/3-1.

\section{APPENDIX A}

In this Appendix we describe the numerical solution of the Percus-Yevick equation for a binary mixture of particles with spherically symmetric interactions of finite range using Baxter's formalism. ${ }^{23}$

Let $e_{\alpha \beta}$ denote the Boltzmann factor of the potential energy between two particles of component $\alpha$ and $\beta, g_{\alpha \beta}$ be the pair correlation function, $h_{\alpha \beta}=g_{\alpha \beta}-1$ be the total correlation function, and $c_{\alpha \beta}$ be the direct correlation function.

Baxter ${ }^{23}$ introduces range parameters $R_{1}$ and $R_{2}$ and associated quantities

$$
\begin{aligned}
& R_{\alpha \beta}=\left(R_{\alpha}+R_{\beta}\right) / 2, \\
& S_{\alpha \beta}=\left(R_{\alpha}-R_{\beta}\right) / 2,
\end{aligned}
$$

such that

$$
c_{\alpha \beta}(r)=0 \text {, if } r>R_{\alpha \beta} .
$$

Then, by introducing new functions $q_{\alpha \beta}$ and their derivatives $q_{\alpha \beta}^{\prime}$ with the boundary conditions

$$
q_{\alpha \beta}(r)=0 \text { for } r \geq R_{\alpha B},
$$

he is able to transform the Ornstein-Zernike relations to

$$
\begin{array}{r}
r c_{\alpha \beta}(|r|)=-q_{\alpha \beta}^{\prime}(r)+2 \pi \rho \sum_{\gamma} x_{\gamma} \int d t q_{\gamma \alpha}(t) q_{\gamma \beta}^{\prime}(r+t) \\
\text { for } S_{\alpha \beta}<r<R_{\alpha \beta},
\end{array}
$$

where the integration with respect to $t$ is over the range

$$
S_{\gamma \alpha}<t<\min \left[R_{r \alpha}, R_{r \beta}-r\right] \text {, }
$$

and

$$
\begin{aligned}
r h_{\alpha \beta}(|r|)= & -q_{\alpha \beta}^{\prime}(r)+2 \pi \rho \sum_{\gamma} x_{\gamma} \int d t q_{\alpha r}(t)(r-t) \\
& \times h_{\gamma \beta}(|r-t|) \text { for } S_{\alpha \beta}<r,
\end{aligned}
$$

where the integration with respect to $t$ is over the range

$$
S_{\alpha_{\gamma}}<t<R_{\alpha_{\gamma}} \text {. }
$$

In our case the Eqs. (A5) and (A6) are closed by the Percus-Yevick approximation ${ }^{22}$

$$
g_{\alpha \beta}=e_{\alpha \beta}\left(g_{\alpha \beta}-c_{\alpha \beta}\right) \text {. }
$$

Baxter's formulation offers the advantage that for finite range potentials the set of Eqs. (A5)-(A7) for $h_{\alpha \beta}$, $c_{\alpha \beta}$, and $q_{\alpha \beta}$ can be solved in appropriate finite range intervals. Having found the solutions in those intervals, the total correlation function can be continued to higher $r$ values by using Eq. (A6).

In our case the interaction potentials $\Phi_{A A}, \Phi_{A B}$, and $\Phi_{\mathrm{BB}}$ shall have finite ranges denoted by $r_{\mathrm{AA}}, r_{\mathrm{AB}}$, and $r_{\mathrm{BB}}$ such that $r_{\mathrm{AA}}<r_{\mathrm{AB}}<r_{\mathrm{BB}}$. In order to keep the integration intervals as small as possible we chose Baxter's range parameters as $R_{2}=r_{\mathrm{BB}}$ and $R_{1}=2 r_{\mathrm{AB}}-r_{\mathrm{BB}}$ if $r_{\mathrm{AB}}$ $>\left(r_{\mathrm{AA}}+r_{\mathrm{BB}}\right) / 2$ or $R_{1}=r_{\mathrm{AA}}$ if $r_{\mathrm{AB}}<\left(r_{\mathrm{AA}}+r_{\mathrm{BB}}\right) / 2$. We checked that a change of the range parameters to somewhat larger values affects the numerical result for the correlation functions only insignificantly.

The set of Eqs. (A5)-(A7) is solved iteratively with $r$ bounded by $R_{\alpha \beta}$ in Eq. (A6). For the numerical evaluation we take $R_{2}$ as unit length and subdivide the interval $\left[-R_{2}, R_{2}\right]$ usually into 200 intervals of equal length $\Delta$. This yields the grid points at which the functions are given numerically. In principle, all the integrations are made with Simpson's rule. In case an integration interval is not bounded by grid points the functions are interpolated or extrapolated by parabolas and the appropriate area under the parabola is calculated.

In solving Eqs. (A5)-(A7) let the functions $g_{\alpha \beta}^{i}$ be the $i$ th approximation. In an inner iteration cycle described below we solve Eq. (A6) for the functions $\left(q_{\alpha \beta}^{i}\right)^{\prime}$ and their integrals $q_{\alpha \beta}^{i}$ which are functionals of the given $h_{\alpha \beta}^{i}$ func- 
tions $\left(h_{\alpha \beta}^{i}=g_{\alpha \beta}^{i}-1\right)$. These $q^{\prime}$ and $q$ functions are inserted into Eq. (A5) which yields the functions $c_{\alpha \beta}^{i}$. Then, from Eq. (A7) we get resulting $g$ functions as

$$
g_{\alpha \beta}^{i, r \beta B}=e_{\alpha B}\left(g_{\alpha B}^{i}-c_{\alpha B}^{i}\right) \text {. }
$$

For constructing the $(i+1)$ th approximation we mix the previous input and output functions getting intermediate functions

$$
g_{\alpha \beta}^{i+1, i n t}=(1-\lambda) g_{\alpha \beta}^{i}+\lambda g_{\alpha \beta}^{i, r \approx s}
$$

with weight factors $\lambda$ of about 0.1. To ensure that $g_{A B}$ $=g_{\mathrm{BA}}$ we finally put

$$
g_{\alpha \beta}^{i+1}=\left(g_{\alpha \beta}^{i+1, i n t}+g_{\beta \alpha}^{i+1, i n t}\right) / 2 .
$$

As a first approximation for $g$ the Boltzmann factors are taken

$$
g_{\alpha \beta}^{1}=e_{\alpha \beta} .
$$

To measure the convergence of the $g$ iteration we use the quantity

$$
\epsilon=4 \sum_{\alpha, \beta} x_{\alpha} x_{\beta} \int_{0}^{R_{\alpha \beta}}\left(g_{\alpha \beta}^{i, r \beta}-g_{\alpha \beta}^{i}\right)^{2} d r .
$$

In general, $\epsilon$ has a lower bound by the fact that the mixed pair correlation functions $g_{A B}^{i, r}$ and $g_{B A}^{i, r e s}$ are calculated in different ways. Due to the numerical treatment these functions never coincide exactly and it is essentially their difference that determines $\epsilon$. In general, $\epsilon$ can be made less than $10^{-8}$. Then, the largest difference between $g_{A B}^{i}$ and $g_{A B}^{i, r e s}$ which occurs close to the maximum of these functions is less than $5 \times 10^{-4}$. This means that the functions $g_{\mathrm{AB}}$ and $g_{\mathrm{BA}}$ always differ by less than $10^{-3}$. We think that this difference is characteristic for the accuracy of all our numerically obtained $g$ functions.

In the inner iteration cycle Eq. (A6) is solved for the functions $\left(q_{\alpha \beta}^{i}\right)^{\prime}$ and $q_{\alpha \beta}^{k}$ with given functions $h_{\alpha \beta}^{i}$. Let the functions $\left(q_{\alpha \beta}^{i k}\right)^{\prime}$ be the $k$ th approximation. These functions are integrated using the boundary condition $\mathrm{Eq}$. (A4) to give $q_{\alpha \beta}^{i k}$. Inserting the $q$ functions into Eq. (A6) we get as next approximation

$$
\left(q^{i, k+1}\right)^{\prime}=-r h^{i}+2 \pi \rho \sum x \int d t q^{i k} h^{i}(r-t)
$$

The starting functions are

$$
\left(q^{i, 1}\right)^{\prime}=-r h^{1}=-r(e-1) \text { for } i=1,
$$

and

$$
\left(q^{i, 1}\right)^{\prime}=\left(q^{i-1, i}\right)^{\prime} \text { for } i>1,
$$

where $l$ refers to the last inner iteration cycle corresponding to the functions $h_{\alpha \beta}^{i-1}$. As a measure of the convergence we take

$$
\delta=\sum_{\alpha, \beta} \int_{s_{\alpha \beta}}^{R_{\alpha \beta}}\left(q_{\alpha \beta}^{i, k+1}-q_{\alpha \beta}^{i, k}\right)^{2} d r .
$$

As it is economic to couple the convergence of the inner iteration cycle to the convergence of the $g$ iteration, the $q$ iteration was stopped if $\delta<10^{-2} \epsilon$.

Having found the solution for $h_{\alpha \beta}$ in the range $0 \leq r$ $\leq R_{\alpha \beta}$ and for $q_{\alpha \beta}$ and $c_{\alpha \beta}$ from Eqs. (A5)-(A7), we continue the functions $h_{\alpha \beta}$ to larger $r$ values by using the appropriate form of $\mathrm{Eq}$. (A6):

$r h_{\alpha \beta}(r)=2 \pi \rho \sum_{\gamma} x_{\gamma} \int_{s_{\alpha \gamma}}^{R_{\alpha \gamma}} d t q_{\alpha \gamma}(t)(r-t) h_{\gamma \beta}(r-t)$.

Whereas hitherto all the functions were given at the grid points $r=k \Delta(k=0, \pm 1, \ldots)$ it is now convenient to give values for $q_{\alpha \beta}$ at $S_{\alpha \beta}+l \Delta(l=0,1, \ldots)$, while the $h_{\alpha \beta}$ shall be given at $R_{\alpha \beta}+m \Delta(m=0, \pm 1, \ldots)$. This change in the grid points was done by cubic interpolation. Now, suppose we know all $h_{\alpha \beta}$ functions up to the grid points $R_{\alpha \beta}+(m-1) \Delta, m$ being the same for all $\alpha \beta$, and ask for $h_{\alpha \beta}$ at the points $R_{\alpha \beta}+m \Delta$. If the integrals in Eq. (A8) are evaluated again with Simpson's rule using $t=S_{\alpha \beta}$ $+l \Delta(l=0,1, \ldots)$ as lattice points then they write as the following algebraic expressions:

$$
\begin{aligned}
\int_{s_{\alpha \beta}}^{R_{\alpha \beta}} d t q_{\alpha \gamma}(t)(r-t) h_{\gamma \beta}(r-t) \\
=\frac{\Delta}{3} q_{\alpha \gamma}\left(S_{\alpha \gamma}\right)\left(R_{\gamma \beta}+m \Delta\right) h_{\gamma \beta}\left(R_{\gamma \beta}+m \Delta\right)+\frac{4 \Delta}{3} q_{\alpha \gamma}\left(S_{\alpha \gamma}+\Delta\right) \\
\quad \times\left[R_{\gamma \beta}+(m-1) \Delta\right] h_{\gamma \beta}\left[R_{\gamma \beta}+(m-1) \Delta\right]+\cdots .
\end{aligned}
$$

Here, only the first term contains the unknown value $h_{\gamma \beta}\left(R_{\gamma \beta}+m \Delta\right)$ and may be written as $a_{\alpha \beta \gamma} h_{\gamma \beta}\left(x_{\gamma \beta}\right)$ with the known coefficient $a_{\alpha \beta \gamma}$ and $x_{\gamma \beta}$ being an abbreviation for $R_{\gamma \beta}+m \Delta$. All the other terms are known and their sum may be called $b_{\alpha \beta \gamma}$. Then, Eq. (A8) writes as

$$
x_{\alpha \beta} h_{\alpha \beta}\left(x_{\alpha \beta}\right)=2 \pi \rho \sum_{\gamma} x_{\gamma}\left(a_{\alpha \beta \gamma} h_{\gamma \beta}\left(x_{\gamma \beta}\right)+b_{\alpha \beta \gamma}\right),
$$

which constitutes a system of four linear algebraic equations for the desired quantities $h_{\alpha \beta}\left(R_{\alpha \beta}+m \Delta\right)$. Since already the $4 \times 4$ matrix relation for the $h$ functions represented by $\mathrm{Eq}$. (A8) decomposes into $2 \times 2$ matrices where $h_{\mathrm{AA}}$ is coupled to $h_{\mathrm{BA}}$ and similarly $h_{\mathrm{AB}}$ to $h_{\mathrm{BB}}$, the evaluation of Eq. (A9) simplifies to the solution of two sets of two linear equations. It shall be mentioned that this continuation procedure was stimulated by a previous work of Perram. ${ }^{43}$

\section{APPENDIX B}

In this Appendix we describe the numerical evaluation of the blip integrals $B_{\alpha \beta}$ given in Eq. (12). By using the definition of $H \hat{y}_{\alpha \beta}$ they can be rewritten as

$$
B_{\alpha \beta}=\int\left(\left\langle e_{\alpha \beta}^{H}\right\rangle /\left\langle e_{\alpha \beta}^{0}\right\rangle\right) \hat{g}_{\alpha \beta} d \mathbf{r}-\int \hat{g}_{\alpha \beta} d \mathbf{r} .
$$

We remind that the angular brackets denote angle-averaging over the orientations $\omega_{1}$ and $\omega_{2}$, if the potential depends on them.

The angle-averaging over $e_{\alpha \beta}^{0}$ does not present any specific numerical problems, as the $u_{\alpha s}^{0}$ potentials are continuous. For the integration corresponding to the dumbbell-dumbbell interaction, e.g., three angles $\theta_{1}$, $\theta_{2}$, and $\phi_{12}$ were introduced, as in Fig. 2(b), and Simpson's rule was applied using 12 subintervals between 0 and $\pi / 2$ for each angle.

The angle-averaging over the Boltzmann factors of the hard interaction potentials needs more care as these are discontinuous. We have to distinguish between the three types of hard interactions. 


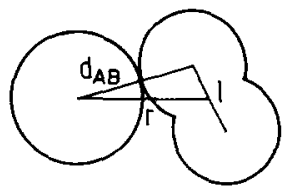

a)

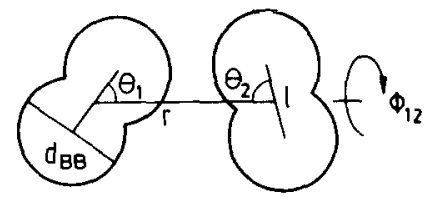

b)
FIG. 2. (a) The sphere/dumbbell, and (b) the dumbbell/ dumbbell geometry.

(a) The interaction between two spheres of diameter $d_{\mathrm{AA}}$. Trivially,

$$
\left\langle e_{\mathrm{AA}}^{\mu}\right\rangle=\theta\left(r-d_{\mathrm{AA}}\right),
$$

where $\theta$ is the Heavyside step function, which is discontinuous at $d_{\mathrm{AA}}$.

(b) The interaction between a sphere and a dumbbell, for which the geometry is shown in Fig. 2(a). After some simple geometric considerations we get

$$
\left\langle e_{\mathrm{AB}}^{H}\right\rangle=\left(r^{2}-d_{\mathrm{AB}}^{2}+l^{2} / 4\right) / r l \text { for } r_{01} \leq r \leq r_{0-},
$$

where

$$
\begin{aligned}
& r_{01}=\left(d_{A B}^{2}-l^{2} / 4\right)^{1 / 2}, \\
& r_{0-}=d_{A B}+l / 2 .
\end{aligned}
$$

The function $\left\langle e_{A B}^{H}\right\rangle$ is now continuous, but its first derivative jumps at $r_{01}$ and $r_{0=}$.

(c) The interaction between two dumbbells, for which the geometry is shown in Fig. 2(b). At a given distance $r$ we need to consider for symmetry reasons only orientations with $0 \leq \phi \leq \pi, 0 \leq \cos \theta_{1} \leq 1$, and $0 \leq \cos \theta_{2}$ $\leq \cos \theta_{1}$. It is convenient to introduce three distances $r_{+}, r_{1}$, and $r_{--}$which are the distances of closest approach for crossed, perpendicular and line-on-line configuration of the two dumbbells

$$
\begin{aligned}
& r_{+}=\left(d_{\mathrm{BB}}^{2}-l^{2} / 2\right)^{1 / 2}, \\
& r_{\perp}=\left(d_{\mathrm{BB}}^{2}-l^{2} / 4\right)^{1 / 2}+l / 2, \\
& r_{--}=d_{\mathrm{BB}}+l .
\end{aligned}
$$

For distances $r$ between $r_{1}$ and $r_{-.,}$one sphere of dumbbell (1) can come into contact only with one sphere of dumbbell (2). There it is possible to obtain from geometric considerations ${ }^{44}$

$$
\begin{aligned}
& \left\langle e_{\mathrm{BB}}^{H}\right\rangle=1-2\left(r-l-d_{\mathrm{BB}}\right)^{2}\left(r-l+2 d_{\mathrm{BB}}\right) / 3 l^{2} r \\
& \qquad \text { for } r_{\perp} \leq r \leq r_{-.} .
\end{aligned}
$$

In the interval $r_{+} \leq r \leq r_{\perp}$ the situation is more complicated and no closed analytical expression has been given hitherto. Therefore, we prescribed a regular lattice in the $\left(\cos \theta_{1}, \cos \theta_{2}\right)$ plane with 200 intervals between 0 and 1. For given values of $\cos \theta_{1}$ and $\cos \theta_{2}$ one can calculate the values $\phi_{s}$ and $\phi_{b}$ at which two spheres stop or begin to overlap. Thus, at least the $\phi$ integration can be performed analytically. As far as numerical results allow conclusions, the function $\left\langle e_{B B}^{H}\right\rangle$ and its first derivative are continuous everywhere, but its second derivative has discontinuities at $r_{+}, r_{\perp}$, and $r_{-. .}$This makes the interpolation procedure of Ref. 21 somewhat questionable.
The $r$ integration in Eq. (B1) does not present serious problems. In principle it is done with Simpson's rule. In the first integral, the lower limit of integration is $d_{\mathrm{AA}}, r_{01}$, or $r_{*}$, respectively. Moreover, in the first integral of $B_{\mathrm{AB}}$ one has to take account also of the discontinuity in the first derivative of $\left\langle e_{A B}^{H}\right\rangle$ at $r_{0-}$.

${ }^{1}$ D. Henderson and P. J. Leonard, in Physical Chemistry-An Advanced Treatise, edited by D. Henderson (Academic, New York, 1971), Vol. 8B, Chap. 7.

${ }^{2}$ I. R. McDonald, Specialist Periodical Report, Statistical Mechanics I, edited by K. Singer (Chemical Society, London, 1973).

${ }^{3}$ J. V. L. Singer and K. Singer, Mol. Phys. 24, 357 (1972).

${ }^{4}$ I. R. MeDonald, Mol. Phys. 23, 41 (1972).

${ }^{5}$ G. A. Mansoori and T. W. Leland, J. Chem. Phys. 53, $1931(1970)$

${ }^{6}$ E. W. Grundke, D. Henderson, J. A. Barker, and P. J. Leonard, Mol. Phys. 25, 883 (1973).

${ }^{7} \mathrm{~J}$. D. Weeks, D. Chandler, and H. C. Andersen, J. Chem. Phys. 54, 5237 (1971); H. C. Andersen, J. D. Weeks, and D. Chandler, Phys. Rev. A 4, 1597 (1971).

${ }^{8}$ L. L. Lee and D. Levesque, Mol. Phys. 26, 1351 (1973).

${ }^{9} \mathrm{~T}$. Boublík, Coll. Czechoslavak. Chem. Commun. 38, 3694 (1973).

${ }^{10} \mathrm{~F}$. Kohler, J. Fischer, and E. Wilhelm, J. Mol. Struct. 84, 245 (1982).

${ }^{11}$ M. Diaz Pena, C. Pando, and J. A. R. Renuncio, J. Chem. Phys. 76, 325, 333 (1982).

${ }^{12} \mathrm{~S}$. Lago, F. Kohler, and J. Fischer (to be published).

${ }^{13}$ G. Stell, J. C. Rasaiah, and H. Narang, Mol. Phys, 27, 1393 (1974).

${ }^{14}$ W. R. Smith, Faraday Discuss. Chem. Soc. 66, 164 (1978).

${ }^{15} \mathrm{~K}$. E. Gubbins and H. C. Twu, Chem. Eng. Sci. 33, 863 (1978), and references therein.

${ }^{16}$ E. Enciso and M. Lombardero, Mol. Phys. 44, 725 (1981).

${ }^{17} \mathrm{D}$. Chandler and H. C. Andersen, J. Chem. Phys. 57, 1930 (1972); D. Chandler, ibid. 59, 2742 (1973).

${ }^{18} \mathrm{M}$. Lombardero and E. Enciso, J. Chem. Phys. 74, 1357 (1981).

${ }^{19} \mathrm{E}$. Enciso, P. Sevilla, and D. J. Tildesley, Second International Conference of Thermodynamic Solutions for NonElectrolytes, Lisbon, 1982.

${ }^{20}$ J. Fischer, J. Chem. Phys. 72, 5371 (1980).

${ }^{21}$ F. Kohler, N. Quirke, and J. W. Perram, J. Chem. Phys. 71,4128 (1979).

${ }^{22}$ J. K. Percus and G. J. Yevick, Phys. Rev. 110, 1 (1958).

${ }^{23}$ R. J. Baxter, J. Chem. Phys. 52, 4559 (1970).

${ }^{24}$ T. Boublik, J. Chem. Phys. 63, 4084 (1975).

${ }^{25} \mathrm{~J}$. A. Barker and D. Henderson, Rev. Mod. Phys. 48, 587 (1976).

${ }^{26}$ W. A. Steele and S. I. Sandler, J. Chem. Phys. 61, 1315 (1974).

${ }^{27}$ T. Boublik and I. Nezbeda, Chem. Phys. Lett. 46, 315 (1977).

${ }^{28}$ L. Verlet, Phys. Rev. 159, 98 (1967); data corrected by D. Levesque and L. Verlet, ibid. 182, 307 (1969).

${ }^{29}$ I. R. McDonald and K. Singer, Mol. Phys. 23, 29 (1972); for errata see Mol. Phys. 24, 464 (1972).

${ }^{30}$ D. J. Adams, Mol. Phys. 32, 647 (1976).

${ }^{3 \mathrm{t}} \mathrm{J}$. P. Hansen and L. Verlet, Phys. Rev. 184, 151 (1969).

${ }^{32}$ K. Nakanishi, S. Okazaki, K. Tkari, T. Higuchi, and H. Tanaka, J. Chem. phys. 76, 629 (1982).

${ }^{33} \mathrm{~J}$. G. Powles and K. E. Gubbins, Chem. Phys. Lett. 38, 405 (1976).

${ }^{34}$ IUPAC-International Thermodynamic Tables of the Fluid State, Argon, 1971, edited by S. Angus and B. Armstrong (Butterworths, London, 1972). 
${ }^{35}$ IUPAC-International Thermodynamic Tables of the Fluid State, Nitrogen, 1977, edited by S. Angus, K. M. de Reuck, and B. Armstrong (Pergamon, New York, 1979).

${ }^{36} \mathrm{~W}$. Pentermann and W. Wagner, J. Chem. Thermodyn. 10, 1161 (1978); W. Wagner, J. Ewers, and W. Pentermann, ibid. 8, 1049 (1976).

${ }^{37}$ R. A. H. Pool, G. Saville, T. M. Herrington, B. D. C. Shields, and L. A. K. Staveley, Trans. Faraday Soc. 58, 1692 (1962).

${ }^{38}$ F. B. Sprow and J. M. Prausnitz, AIChE J. 12, 780 (1966).

${ }^{39}$ K. L. Lewis and L. A. K. Staveley, J. Chem. Thermodyn.
7, 855 (1975); K. L. Lewis, G. Saville, and L. A. K. Staveley, ibid. 7, 389 (1975).

${ }^{40} \mathrm{~J}$. S. Rowlinson and F. L. Swinton, Liquids and Liquid Mixtures (Butterworths, London, 1982), Chaps. 5 and 8.

${ }^{41}$ T. W. Leland, J. S. Rowlinson, and G. A. Sather, Trans. Faraday Soc. 64, 1447 (1968).

${ }^{42}$ N. S. Snider and T. M. Herrington, J. Chem. Phys. 47, 2248 (1967).

${ }^{43}$ J. W. Perram, Mol. Phys. 30, 1505 (1975).

${ }^{44} \mathrm{H}$. Oelschlager, Institute of Physical Chemistry, University of Vienna (unpublished). 